\title{
Labyrinthe
}

\section{The Walking Dead\&nbsp : oubli, rumination et emblèmes du temps}

(De l'utilité et des inconvénients des séries pour la non-vie)

\section{Anne Bourse}

\section{OpenEdition}

\section{Journals}

Édition électronique

URL : http://journals.openedition.org/labyrinthe/4198

DOI : $10.4000 /$ labyrinthe.4198

ISSN : 1950-6031

Éditeur

Hermann

Édition imprimée

Date de publication : 15 août 2011

Pagination : 91-99

ISBN : 9782705681470

Référence électronique

Anne Bourse, "The Walking Dead\&nbsp : oubli, rumination et emblèmes du temps », Labyrinthe [En ligne], 37 | 2011 (2), mis en ligne le 01 août 2013, consulté le 20 avril 2019. URL : http:// journals.openedition.org/labyrinthe/4198; DOI : 10.4000/labyrinthe.4198

Propriété intellectuelle 


\title{
The Walking Dead: oubli, rumination et emblèmes du temps
}

(De l'utilité et des inconvénients des séries pour la non-vie)

Anne Bourse

\begin{abstract}
« Nous ne voulons servir l'histoire que dans la mesure où elle sert la vie [...]. Quant à savoir jusqu'à quel point la vie a besoin des services de l'histoire, c'est là une des questions et des inquiétudes les plus graves concernant la santé d'un peuple, d'un individu, d'une civilisation. Car trop d'histoire ébranle et fait dégénérer la vie, et cette dégénérescence finit également par mettre en péril l'histoire elle-même. »"
\end{abstract}

Nietzsche, Deuxième Considération inactuelle.

La récente création de Frank Darabont, The Walking Dead, diffusée en octobre 2010 sur la chaîne câblée AMC, rassemble toutes les caractéristiques de la production télévisée contemporaine: esthétique cinématographique, découpage feuilletonesque et temporalité immersive, elle porte non seulement au jour les symptômes et les contradictions d'un monde en crise, saturé d'images, de marchandises et de mémoires concurrentes, mais elle met plus précisément en abyme les effets de sidération mentale et d'annexion sociale qu'exerce la fiction sur la communauté médusée des spectateurs. Venant défier les grands romans de l'excès, ceux dont l'ambition fut notamment d'englober une durée historique dans sa totalité et dont la publication s'est effectivement répartie sur de nombreuses années ${ }^{1}$, la série télé du début du $\mathrm{XXI}^{\mathrm{e}}$ siècle se fait monstrum, au sens étymologique du terme: elle révèle, par accumulation, les signes de la catastrophe et nous avertit de son propre pouvoir de fascination.

1. Voir les analyses de Tiphaine Samoyault sur le roman-fleuve dans Excès du roman, Paris, Maurice Nadeau, 1999, p. 51-110. 
Adaptée de l'excellent comic-fleuve de Robert Kirkman², The Walking Dead retrace les aventures d'un petit groupe de survivants au sein d'un monde dévasté et peuplé de zombies à la suite d'une apocalypse qui demeure inexpliquée. Depuis les années soixante et les trois films fondateurs du genre (The Last Man on Hearth de Ubaldo Ragona (première adaptation du roman de Richard Matheson, I Am Legend), Dead of Night (Le Mort-vivant) de Bob Clark et Night of the Living Dead de George Romero), le personnage du mort-vivant supplée régulièrement aux fantômes et aux vampires de la tradition gothique comme allégorie antiromantique de l'aliénation et comme témoin privilégié de notre société de surproduction. Comme l'a fort bien rappelé une récente programmation de l'Auditorium du Louvre consacrée aux « figures contemporaines de la non-mort », le zombie se distingue par son caractère de rebut urbain, foncièrement prolétarien: « figure de malédiction dans l'univers colonial esclavagiste comme dans le monde capitaliste néo-libéral, [il] donne à voir et à penser une histoire matérielle de la privation de soi qui a partie liée avec la révolution industrielle, depuis le travail à la chaîne du fordisme jusqu'aux actuelles délocalisations ${ }^{3}$ ». Ce mort qui n'en finit pas de revenir en masse, déchet aux râles indolents et à l'agonie infinitésimale, arpente la terre sans relâche avec sa cohorte de semblables interchangeables; leur maigre énergie n'est plus celle d'un désespoir exalté mais bien du triste et rudimentaire instinct de consommation ${ }^{4}$.

Bien qu'elle ne compte pour l'instant que six épisodes d'environ une heure chacun ${ }^{5}$, The Walking Dead nous confronte à quelques problèmes essentiels, tel que l'entrecroisement de temporalités hétérogènes au sein d'une même durée anxiogène. Comme le montrent les nombreuses scènes de «transformation » (le passage, à la faveur d'une morsure

\footnotetext{
2. The Walking Dead est d'abord une série de comic books américains en noir et blanc, publiée mensuellement par Image Comics depuis 2003, et toujours en cours. Robert Kirkman en est le scénariste, Tony Moore et Charlie Adlard les deux dessinateurs. Les 78 volumes originaux sont publiés en France par Delcourt sous la forme de 13 tomes.

3. Marcella Lista, "Survivances: figures contemporaines de la non-mort », texte de présentation de la programmation thématique Revenants. Images, figures et récits du retour des morts, organisée à l'Auditorium du Louvre du 13 janvier au 28 mars 2011.

4. On se souvient que dans le deuxième volet de la trilogie de George Romero, Zombie (1978), vivants et morts-vivants convergent ironiquement vers cet autre symbole du consumérisme américain qu'est le mall, le centre commercial, temple du conditionnement grégaire et de la désindividuation.

5. Le tournage des treize épisodes de la deuxième saison a débuté en juin 2011.
} 
incurable, du statut d'encore-vivant à celui de pas-tout-à-fait-mort) qui ponctuent la série à la manière d'un sinistre compte à rebours, les zombies apparaissent comme les éléments surnuméraires d'un passé trop proche pour être correctement digéré et devenir réellement passé, réduisant ainsi toute appréhension de l'avenir à la logistique précaire d'un présent vécu perpétuellement aux aguets, en attendant de venir grossir à son tour la horde des charognes ambulantes. Si elle ne nous permet pas pour le moment de savoir à quoi ressemblerait la chronique d'un univers entièrement contaminé et dont les zombies seraient les seuls protagonistes, The Walking Dead évoque néanmoins à grand renfort d'effets spéciaux la matérialité de cette très lente survivance que rend visible le corps affligé du zombie. À rebours de la sensualité prédatrice du vampire, le mort-vivant dégoûte en effet par sa gaucherie pathétique et la décomposition de sa chair, faisant justice aux remarques de Walter Benjamin dans l'Origine du drame baroque allemand sur la vitalité du cadavre comme " emblème » de l'écoulement du temps: "Vue sous l'angle de la mort, la vie est production de cadavres [...]. Et ce n'est pas un hasard si les ongles et les cheveux, et précisément eux, dont le corps vivant se débarrasse comme de choses mortes, continuent à pousser sur le cadavre. Il y a dans la physis, dans la mnémè elle-même un memento mori qui reste en éveil ${ }^{6} \gg$.

C'est que la série de Frank Darabont traite avant tout de l'expérience de la survie à l'échelle collective (la menace zombie comme forme extrême de la robinsonnade moderne) et de la relation qu'entretient le vivant avec son environnement, dans ce qu'il peut avoir à la fois de plus familier et de plus violent, de plus morne et de plus extraordinaire, de plus répétitif et de plus singulier. Contagion, prolifération, désubjectivation, étrangement du monde connu et de tout lien humain (amical, social, familial, amoureux), The Walking Dead met frontalement en scène notre

\footnotetext{
6. Walter Benjamin, Origine du drame baroque allemand, trad. Sybille Muller, Paris, Flammarion, «Champs », 1985, p. 235. Je renvoie également au livre que Georges Didi-Huberman a consacré à Aby Warburg et au concept de « survivance ", où l'on trouve cette belle définition du symptôme, applicable à la paradoxale figure du zombie: «Qu'est-ce, enfin, que ce moment où viennent débattre et s'entrelacer le présent du pathos et le passé de la survivance, l'image du corps et le signifiant du langage, l'exubérance de la vie et l'exubérance de la mort, la dépense organique et la convention rituelle, la pantomime burlesque et le geste tragique? Qu'est-ce donc que ce moment, si ce n'est celui du symptôme, cette exception, cette désorientation du corps et de la pensée [...]? » L'Image survivante : Histoire de l'art et temps des fantômes selon Aby Warburg, Paris, Minuit, «Paradoxe », 2002, p. 270.
} 
rapport fonctionnel avec le « milieu ». Simple personnage de fiction ou spectateur confortablement lové dans son canapé, le zombifié témoigne ainsi de la pire des situations d'urgence - situation " catastrophique » au sens que lui confère le neurologiste Kurt Goldstein : celle d'un vivant « commandé du dehors par le milieu » ${ }^{7}$ et voué à répéter compulsivement les mêmes gestes, qu'il s'agisse de la marche hébétée du macchabée, du massacre inlassable des non-morts par les survivants ou du visionnage maniaque de séries par le nolife $^{8}$, prisonnier d'une durée improductive que règle l'insatiable enchainement des épisodes.

À cet égard, le format sériel - de la bande-dessinée comme de la télévision - radicalise très nettement l'ambition du genre cinématographique 9 . Au-delà du canevas hollywoodien des 90 minutes, nécessairement engoncé entre la montée de la crise et sa résolution finale, The Walking Dead offre une épaisseur et une complexité temporelle inespérée au quotidien déliquescent de la survie en terre zombie. Robert Kirkman, dans la postface du deuxième tome de l'édition française de The Walking Dead, évoque

7. Georges Canguilhem, La Connaissance de la vie (1965), Paris, Vrin, « Bibliothèque des textes philosophiques », 2003, p. 188.

8. Désignant à l'origine une personne qui consacre la majeure partie de son temps aux jeux vidéo au détriment de toute vie sociale et affective, cette expression péjorative s'est banalisée et englobe à présent deux types d'« addiction » sociologiquement opposés, ayant trait soit au travail (workaholism) soit à la consommation excessive de produits culturels tels que les mangas, les jeux en ligne, les films ou les séries télé. En ce sens commun, le stéréotype du nolife contribue à la dépréciation générale de la fiction dérivée du sempiternel débat philosophique entre « réel » et « virtuel »: seuls le chômeur, le grabataire et le désœuvré peuvent en effet se permettre de « perdre leur temps » en fables imaginaires. Cependant, la série de zombies me semble emprunter une tout autre direction, bien plus benjaminienne que platonicienne. Au-delà de la simple assimilation du spectateur au consommateur abêti, délaissant la problématique rebattue du simulacre et de la fiction trompeuse, The Walking Dead nous rappelle que nous vivons à l'ère de la reproductibilité: à l'instar des œuvres d'art et des images étudiées par Benjamin, les zombies sont conçus «pour être reproductibles » et se reproduisent en série. Loin de jouer le rôle d'inconsistants hologrammes qui permettraient de fustiger l'hypothétique " virtualisation » de notre monde contemporain, ils accentuent au contraire le poids de la matière fictionnelle dont se nourrit notre culture. Comme les films de David Lynch où prospèrent toujours, derrière l'hygiénisme de façade, de répugnants détritus de l'usine à rêves américaine (l'oreille coupée en ouverture de Blue Velvet, le vomi de Lula dans Wild at Heart, le clochard de Mulholland Drive pour n'en donner que quelques exemples), les zombies qui « refoulent» de partout mettent en lumière un problème à la fois politique, écologique et archivistique : celui de la production, de la dépense, du stockage et du recyclage des matières que sont les marchandises, les images, les textes et les souvenirs.

9. Rappelons que The Walking Dead n'est pas la première série de zombies. En 2008, la chaîne britannique E4 a notamment produit la mini-série Dead Set (un épisode de 45 minutes et quatre épisodes de 25 minutes), dont l'action se déroule au sein du studio de l'émission de télé réalité Big Brother, dernier bastion de résistance face au désastre planétaire de la zombification des corps et des cerveaux. 
ainsi l'une de ses sources d'inspiration: «Pour moi, le moment le plus pénible dans un film de zombie, c'est la fin. Je veux toujours savoir ce qu'il se passe ensuite. Même lorsque tous les personnages sont morts [...]. On ne se demandera JAMAIS ce qu'il est arrivé ensuite à Rick [le héros], on y assistera. Walking Dead sera un film de zombie qui ne connaîtra pas de fin ${ }^{10} \gg$. Life goes on after death : pour retarder infiniment la fin, il faut sans cesse renouveler la vie d' « après » le désastre... Tel est le credo de Frank Darabont quand il transpose le comic book puisque tel est précisément l'apanage de la "saison » télévisuelle, routinière et cyclique mais potentiellement illimitée (tant qu'elle maintient ses taux d'audience) et ouverte à l'expérimentation d'innombrables possibles narratifs. Dès les premiers épisodes, une fois posées les prémices de l'intrigue (au sortir d'un long coma, le sheriff Rick Grimes découvre un monde ravagé et part à la recherche de sa famille, qui vit réfugiée avec d'autres rescapés à la lisière d'Atlanta), la version télé de The Walking Dead s'engage ainsi sur des sentiers de traverse, alternant des visions d'horreur très fidèlement reconstituées à partir de la bande-dessinée et la réécriture de certaines séquences clés : le fils, Carl, et l'ami, Shane, échappent par exemple au destin particulièrement traumatique que leur réserve Robert Kirkman dès la clôture du premier tome, tandis que de nouveaux personnages sont librement insérés par Frank Darabont. À la lecture des treize tomes de The Walking Dead, qui s'enfoncent dans une violence physique et mentale de plus en plus insoutenable, on s'interroge notamment sur la manière dont les scénaristes de la série disposeront de cette intéressante marge de manœuvre qu'est l'adaptation pour éviter la censure (ou du moins l'interdiction aux moins de 18 ans) et tout simplement inventer les moyens de redonner à voir et à entendre l'interminable cauchemar de Rick Grimes et de ses compagnons.

La série n'en est qu'à ses débuts mais tient donc en germe la promesse de consigner des années entières de lutte contre la mort, en nous plongeant dans un récit aussi atypique (tous les repères d'une vie normale ayant disparu au profit d'un régime d'exception) qu'itératif (la survie ne s'effectuant qu'au prix de la répétition de gestes et d'affrontements toujours semblables). C'est cette vertigineuse isomorphie entre l'objet sériel et son 
sujet, profondément inscrit dans la durée, nécessairement « en-duré $»^{11}$ sur de longues saisons, qui bouleverse le traitement cinématographique de la problématique zombie. Archi-film d'un siècle à peine entamé qu'il tombe déjà en ruines, The Walking Dead se met ainsi au défi d'étirer le temps à la manière (illisible, colossale, encyclopédique, cannibale, etc.) dont le « roman-monde » (Tiphaine Samoyault) et le « livre-monstre » (Claro) bravent l'identité des lecteurs ainsi que les normes établies:

Le gigantisme d'une œuvre a peut-être également un sens social plus pervers qu'on ne pourrait le croire à première vue, tant il oblige celui qui lit à prendre conscience de la non-valeur accordée par la société au temps de la lecture. Ces fameux livres qu'on dit souvent « illisibles » ne le sont bien souvent que parce qu'ils exigent un temps d'absorption que notre emploi du temps nous refuse. En cela, le livre se pose, et se posera toujours, comme ennemi, rival du temps de travail; il est de l'ordre de la perte, au sens ontologique et économique, car le gain qu'il propose ne saurait être ni quantifié ni assuré, même en négatif ${ }^{12}$.

L'analyse des outrances de la "littératologie » redouble en tous points celles d'une série comme The Walking Dead. Bien qu'il se mesure aisément en nombres d'heures passées devant l'écran, ce temps irrémédiablement " perdu » à différer la fin de l'histoire à la façon dont les derniers représentants de l'espèce combattent pour leur survie témoigne d'un dérèglement, d'un débord à la fois social et existentiel. Car le temps de la fiction est toujours un temps matériellement vécu par le spectateur et le lecteur; excédant la simple durée de vie des personnages, il correspond à notre inscription dans le temps lui-même. On nuancera toutefois la comparaison en précisant que si nous sommes autant « les feuilletons de ce que nous lisons » que de ce que nous regardons, si « la fragmentation de notre propre temps intime décide de la consommation des produits de l'usine ${ }^{13} »$, « l'utopie ${ }^{14} »$ réalisée par la lecture demeure

11. La formule est de Georges Didi-Huberman, « Ninfa Dolorosa, ou les figures de la lamentation », Séminaire EHESS/INHA, années universitaires 2004-2007.

12. Claro, « De la littératologie », dans Le Clavier cannibale, Paris, Inculte, «Temps réel », 2009, p. 45-46. Nous soulignons.

13. Ibid., p. 50.

14. « La lecture n'est pas l'apanage des oisifs ou des bourgeois, comme on l'a dit à l'époque d'une certaine terminologie; c'est avant tout une utopie, celle d'un état où l'apparente passivité dissimule une 
plus valorisée que celle des images de la télévision. À la différence de la noble captivité que nous impose la littérature, l'accoutumance distillée par la série se décline sur un mode mineur, celui de la pop culture, qu'il faut non seulement entendre dans sa trivialité mais aussi dans sa très grande quantité. Face à l'afflux de fictions toujours plus nombreuses, de récits infiniment prolongés, le spectateur de séries télé se retrouve ainsi - en lieu et place du principe de sélection darwinien, tels les héros en sursis de The Walking Dead - amené à «éliminer le pire sans avoir part à la production de nouveaux êtres, normalisés par leur adaptation non préméditée à de nouvelles conditions d'existence, la monstruosité devenant règle et l'originalité banalité provisoire ${ }^{15} »$.

Et c'est précisément en vertu de sa routine, de son conformisme scénaristique et de ses échecs mêmes (les derniers épisodes de la saison 1 déçoivent par un final paresseusement calqué sur celui de trop nombreux films du genre), qu'une série comme The Walking Dead permet enfin d'aborder le lien qui unit le « milieu » à l'apparition de nouvelles formes (de vie, d'art). La figure du zombie joue ici le rôle de paradigme ultime. Situé à la trouble frontière de la mort (la certitude d'une fin inconditionnelle) et de la monstruosité (l'inquiétude d'un organisme faisant écart à la norme), il oppose une double menace à la "formation de forme », pour reprendre l'expression de Georges Canguilhem: non-vivant, le zombie est la « contre-valeur vitale » qui borne implacablement le vivant de l'extérieur; non-viable, il en est aussi l'intime négation, « la menace accidentelle et conditionnelle d'inachèvement ou de distorsion ${ }^{16} »$. Élire le mort-vivant comme sujet de création audio-visuelle ne peut donc manquer d'affecter le protocole de la représentation, la construction des épisodes, la durée de vie même de la série. De manière comparable à Breaking Bad, dont le héros est atteint d'un cancer en phase terminale, The Walking Dead se voue (au moins théoriquement) à l'exploration des limites, qu'elles soient celles de la mort (par quelles ruses narratives la repousser le plus longtemps possible? la série peut-elle continuer après la zombification de tous les protagonistes?) ou celles de la difformité:

activité non-productive [...], porteuse d'un projet autrement plus ambitieux : remettre en cause la notion de lecture telle que nous la concevons ordinairement, c'est-à-dire en termes de linéarité. » Ibid., p. 46. 15. Georges Canguilhem, op. cit., p. 176.

16. Ibid., p. 221. 
quel est le degré de violence, de désespoir, d'insolite et de pathologique que créateurs et spectateurs (sans oublier les producteurs de la série) accepteront de supporter? De ce point de vue, les six premiers épisodes ne font pas tant infraction aux codes qu'ils ne reconduisent le mouvement positiviste de domestication du monstrueux, par lequel « l'irrégulier est rendu à la règle, le prodige à la prévision » et le monstre au « bocal de l'embryologiste où il sert à enseigner la norme ${ }^{17}$ ». Canguilhem a bien montré comment la pensée rationnelle et le regard scientifique s'étaient progressivement substitués à la tératologie fantastique du Moyen Âge et de la Renaissance qui célébraient la monstruosité comme source de l'imagination. Ainsi qu'en témoigne le soin accordé aux maquillages et aux effets numériques chargés de restituer l'horreur, The Walking Dead semble encore hésiter entre ces deux pôles. Les zombies y sont à la fois supports de fascination esthétique et de maîtrise technique, à défaut d'être les inspirateurs d'une forme télévisuelle radicalement inédite.

The Walking Dead est certes bien loin de réinventer l'anatomie conventionnelle de l'écriture et du montage, mais cette série n'en produit pas moins des effets concrets sur nos actuelles «formes de vie». En nous exposant sur une longue durée à l'archétype du « débat » avec un milieu hostile, en adaptant de façon subtile les traits de la bande dessinée à ceux, mouvants et colorés, de la télévision, en régurgitant les indices les plus gore d'un passé contrarié, The Walking Dead nous ouvre à une expérience nietzschéenne contradictoire, qui consiste à oublier sa propre existence tout en perdant pied dans le marécageux « torrent du devenir ${ }^{18}$ » qu'incarne exemplairement le zombie. Battant en brèche les affirmations lapidaires d'un Jean-Luc Godard (« La télévision fabrique de l'oubli, le cinéma fabrique des souvenirs », " pour moi la grande histoire c'est l'histoire du cinéma. Elle est plus grande que les autres parce qu'elle

17. Ibid., p. 228.

18. « Représentez-vous [...] un homme qui ne posséderait pas la force d'oublier et serait condamné à voir en toute chose un devenir : un tel homme ne croirait plus à sa propre existence, ne croirait plus en soi, il verrait tout se dissoudre en une multitude de points mouvants et perdrait pied dans le torrent du devenir [...]. Toute action exige l'oubli, de même que toute vie organique exige non seulement la lumière, mais aussi l'obscurité. » Friedrich Nietzsche, De l'utilité et des inconvénients de l'histoire pour la vie, Considérations inactuelles II, trad. Pierre Rusch, Paris, Gallimard, Folio « Essais », 1990, p. 96-97. Nous soulignons. 
se projette $\left.{ }^{19} »\right)$, la série déroule obstinément la trame d'une mémoire insomniaque, débordant de vies possibles, rêvées, ratées, inachevées, distordues. C'est paradoxalement par son excès de souvenirs et de corps putréfiés que The Walking Dead nous aide à guérir de la « fièvre historienne », nous invitant à ruminer, d'épisodes en épisodes, « toute chose passée, proche ou lointaine [...], et pour ainsi dire [à] la transformer en [notre] propre sang ${ }^{20} »$.

19. Jean-Luc Godard, Histoire(s) du cinéma, Épisode 2a « Seul le cinéma », Paris, Gallimard, 1998, p. 44 et 49 .

20. Friedrich Nietzsche, op. cit., p. 97-98. 